\title{
Controlling Chaos in Damped and Driven Morse Oscillator via Slave-Master Feedback
}

\author{
S. Behnia ${ }^{a, *}$, A. AKHSHANi ${ }^{b, c}$, M. PANAHi $^{b}$ AMd R. ASADI ${ }^{d}$ \\ ${ }^{a}$ Department of Physics, Urmia University of Technology, Orumieh, Iran \\ ${ }^{b}$ Department of Physics, IAU, Orumieh Branch, Orumieh, Iran \\ ${ }^{c}$ School of Physics, Universiti Sains Malaysia, 11800 USM, Penang, Malaysia \\ ${ }^{d}$ Department of Computer, IAU, Orumieh Branch, Orumieh, Iran \\ (Received May 6, 2012; in final form May 17, 2012)
}

\begin{abstract}
The dynamical behavior of the Morse oscillator is investigated primarily by means of the Lyapunov exponent and bifurcation diagrams. Then, the problem of controlling chaos for this oscillator is studied using a new method introduced by Behnia and Akhshani, which is based on the construction of slave-master feedback. In the control model based on slave-master feedback, the oscillator as the slave system is coupled with a dynamical system as the master, so its implementation becomes quite simple and similar statements can be made for the high dimensional cases. The validity of this method is verified by numerical simulations. The obtained results show the effectiveness of the proposed control model.
\end{abstract}

DOI: 10.12693/APhysPolA.123.7

PACS: 05.45.-a, 05.45.Gg, 05.45.Pq

\section{Introduction}

The presence of chaos in nonlinear dynamical systems has been extensively demonstrated. Chaotic phenomenon is likely to be the complex, and it is often undesirable in practice. So, over the past twenty years, greater efforts have been spent to control chaos in various studies [1-9]. There are two ways to control chaos in general. One is to suppress the chaotic dynamical behavior and the other is to generate or enhance chaos in nonlinear systems known as anticontrol of chaos [10, 11].

Nowadays, different techniques and methods have been proposed to achieve chaos control. OGY method [12], differential geometric method [13], feedback and nonfeedback control [14-17], inverse optimal control [18], adaptive control $[19,20]$ and back stepping design technique [21] are some of famous methods presented up to now. The Morse oscillator is the most prominent example of an anharmonic oscillator that has found wide applications. It is one of the most realistic models for describing the vibrations of a diatomic molecule, being interesting not only from the experimental, but also from the theoretical point of view. Also, the Morse oscillator is still a model of the true interatomic potential energy. There are many contributions investigating the Morse oscillator with classical, semiclassical and quantum mechanical methods [22-31]. In particular, Kapral et al. [32] found the amplitude-resonance curves, and the sequence of period-doubling bifurcations leading to chaos and tangent bifurcations associated with period three windows in the chaotic region. Therefore, investigations on the controlling of chaos in this system is of almost importance [33].

Recently, the authors have proposed a new chaos control method based on slave-master feedback (SMF) [34].

The purpose of this paper is to introduce a more ex-

*corresponding author; e-mail: s.behnia@sci.uut.ac.ir plicit approach to the control continuous nonlinear dynamical systems. Because of the tremendous complexity of chaotic dynamics, the problem is restricted to the Morse oscillator which is used as a well-known model for molecular vibrations [35-38]. Despite the simplicity of the Morse oscillator, the dynamical behavior is extremely rich and research on the area is still going on today [33]. By means of the Lyapunov exponents and bifurcation diagrams the dynamics of the system before and after applying the control model is investigated. The validity of the presented method is verified by numerical simulations. The obtained results show that the introduced control model is pretty efficient and easy to implement.

\section{Slave-master feedback control}

The major key ingredient for the control of chaos is the observation that a chaotic set, on which the trajectory of the chaotic process lives [39], has embedded within it a large number of unstable period orbits. Then, the accessibility of the chaotic systems to many different periodic orbits combined with its sensitivity to small perturbations allow for the control and the manipulation of the chaotic process. The OGY approach is then as follows. One first determines some of the unstable periodic orbits that are embedded in the chaotic set. Then examines the location and the stability of these orbits and chooses one which yields the desired system performance. Finally, one applies small control to stabilize this desired periodic orbit.

The controlled process obeys the following state equations:

$$
\dot{x}=F(x, a),
$$

where $x$ is a state variable, the vector-function $\dot{x}=\frac{\mathrm{d} x}{\mathrm{~d} t}$ is assumed to be continuously differentiable and by variable $a$ is meant the changeable system parameter, rather than the standard input control variable.

The present analysis consider the parameter $a$ to be variable in the time such that thoroughly be changed by another chaotic map as it is shown below [40]: 


$$
\dot{a}=\frac{4 a-(1-a)^{2}}{\Delta t(1-a)^{2}},
$$

where $\Delta t$ is an arbitrary number between $(0,1)$.

We can introduce dynamical controlling chaotic processes as follows:

$$
\left\{\begin{array}{l}
\dot{x}=\Phi_{N}(x, \dot{a}), \\
\dot{a}=\frac{4 a-(1-a)^{2}}{\Delta t(1-a)^{2}} .
\end{array}\right.
$$

In this paper we are interested in explaining the introduced control model based on the continuous form of the hierarchy of one-parameter chaotic maps which are introduced in our previous papers [40], where the logistic map is topologically conjugated [41] to the introduced map, Eq. (2). The introduced chaotic maps can be used as dynamical control. The whole results obtained shows the possibility of stabilizing discrete-time systems. Now, the chaos control problem can be studied without requiring any knowledge about the state of system.

\section{Damped and driven Morse oscillator}

The differential equation which describes the damped and driven Morse oscillator (DDMO) system is as follows:

$$
\ddot{x}=-\beta \mathrm{e}^{-\alpha x}\left(1-\mathrm{e}^{-\alpha x}\right)-\gamma y+F \cos (\omega t),
$$

where $\beta, \alpha, \gamma, F$ and $\omega$ are the parameters which can drive the system to chaos. Before applying dynamical control method to the system, it is more convenient to reduce the order of Eq. (1) to first order ordinary differential equations as:

$$
\left\{\begin{array}{l}
\dot{x}=y, \\
\dot{y}=-\beta \mathrm{e}^{-\alpha x}\left(1-\mathrm{e}^{-\alpha x}\right)-\gamma y+F \cos (\omega t) .
\end{array}\right.
$$

This form of DDMO is coupled through one of its control parameters (e.g. $\beta, \alpha, \gamma, F$ and $\omega$ ) with an external dynamical system as a controllable system (Eq. (3)).

In this study, $\gamma$ is selected as a control parameter and Eq. (3) has been applied to it. By considering the Morse oscillator (Eq. (6)) and the control system (Eq. (5)) as a three-dimensional dynamical system the simple model for controlling the stability of the Morse oscillator is introduced as follows:

$$
\left\{\begin{array}{l}
\dot{x}=y \\
\dot{y}=-\beta \mathrm{e}^{-\alpha x}\left(1-\mathrm{e}^{-\alpha x}\right)-\gamma y+F \cos (\omega t), \\
\dot{\gamma}=\frac{4 \gamma-\gamma(1-\gamma)^{2}}{\Delta t(1-\gamma)^{2}}
\end{array}\right.
$$

\section{Result}

The results of the numerical experiments are given to verify the effectiveness of the proposed control approach in this section. Various numerical simulations including bifurcation diagrams and the Lyapunov exponent plots are presented for the two scenarios: (1) the state of the uncontrolled system (see Figs. 1-10) and (2) the state of the system being under control (see Figs. 11-20).

(1) The state of the uncontrolled system: to compare the uncontrolled and controlled behavior of the DDMO system, the uncontrolled behavior of it is presented by means of the bifurcation diagrams and Lyapunov exponent plots. Figures 1-10 show bifurcation diagrams and the Lyapunov exponent plots, associated with the different parameter values and initial conditions. Numerical simulations clearly demonstrate the existence of chaotic behavior in the absence of the control process. As shown in Figs. 1-10 all of parameters $\beta, \alpha, \gamma, F, \omega$ can drive the DDMO to chaos. For instance DDMO has chaotic motion while $\beta=1, \alpha=1, \gamma=0.16, F=0.2$ and $\omega=1$, and has a stable behavior while $\beta=0.89, \alpha=1, \gamma=0.8, F=2.5$ and $\omega=\sqrt{3}$. A detailed review about these parameters and chaotic regions of DDMO system is presented in [33].

(2) The state of the system being under control: bifurcation diagrams and the Lyapunov exponent plots of the DDMO in the presence of control process are shown in Figs. 11-20. Having a look at the figures it can be observed that the spectrum of the Lyapunov exponents and the bifurcation diagrams confirm that the chaotic behavior of the DDMO can be either fully (see Figs. 12, 14, 16, 18, 20) or regionally (see Figs. $11,13,15,17,19)$ controlled. In the Lyapunov exponent plots, the control process has been applied to the system from the beginning iteration. To consider the chaotic finite domain of the system in the bifurcation diagrams, the control process is applied to the system after it is driven to fully chaotic regions (see Figs 11, 13, 15, 17, 19).

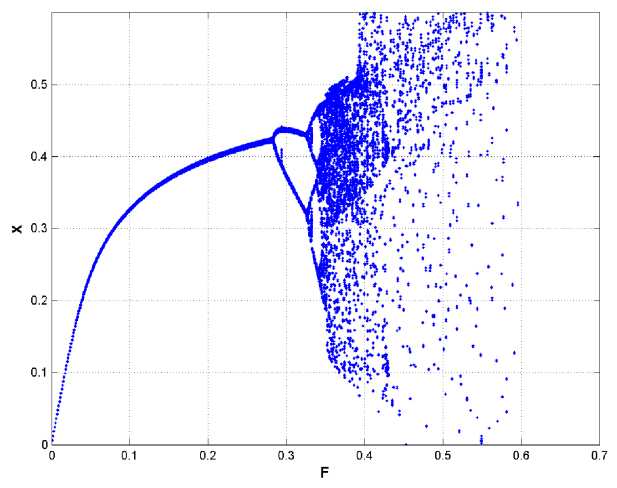

Fig. 1. Bifurcation diagram of uncontrolled evolution of system, where $\gamma=0.16, \quad \beta=1, \omega=\sqrt{1}$, $\alpha=1,[x(0), y(0)]=[0.001,0.002]$.

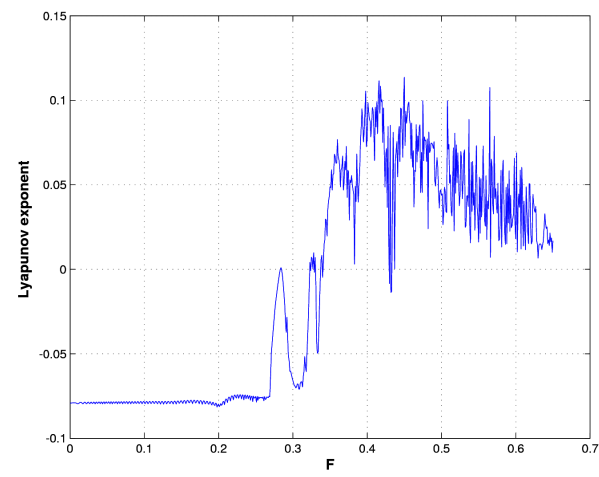

Fig. 2. Lyapunov exponent of uncontrolled evolution of system, where $\gamma=0.16, \beta=1, \omega=\sqrt{1}$, $\alpha=1,[x(0), y(0)]=[0.001,0.002]$. 


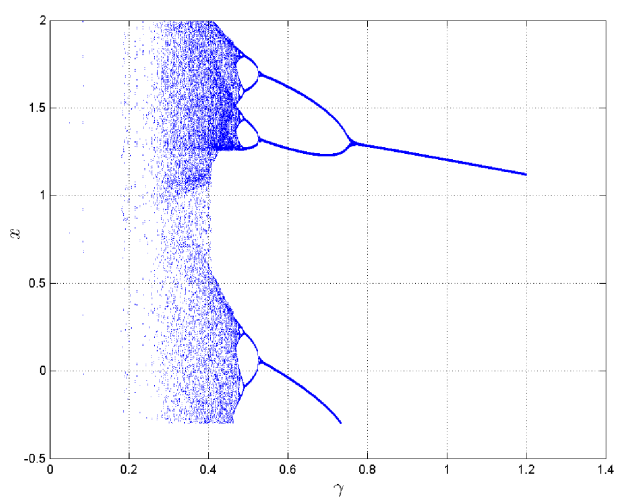

Fig. 3. Bifurcation diagram of uncontrolled evolution of system, where $F=2.5, \beta=0.89, \omega=\sqrt{3}$, $\alpha=1,[x(0), y(0)]=[0,0.5]$.

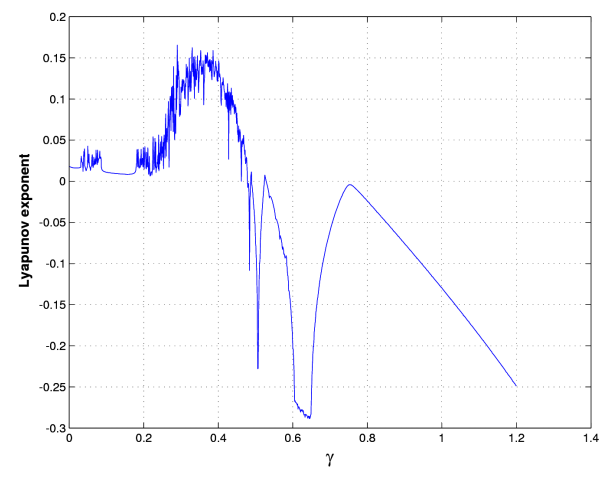

Fig. 4. Lyapunov exponent of uncontrolled evolution of system, where $F=2.5, \quad \beta=0.89, \omega=\sqrt{3}$, $\alpha=1,[x(0), y(0)]=[0,0.5]$.

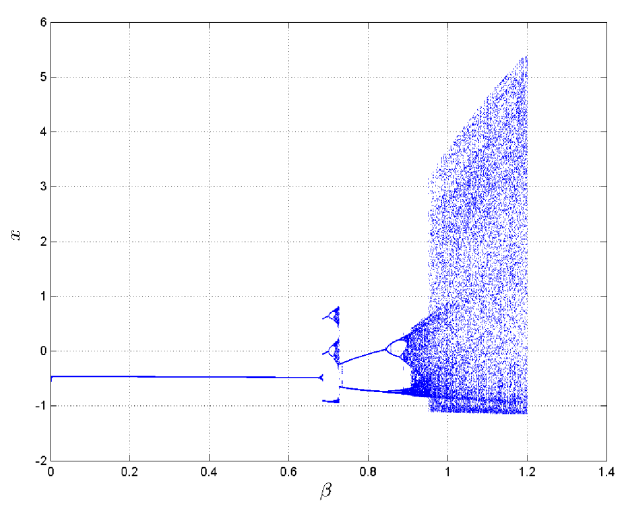

Fig. 5. Bifurcation diagram of uncontrolled evolution of system, where $F=2.5, \gamma=0.3, \omega=\sqrt{3}$, $\alpha=0.8,[x(0), y(0)]=[0.001,0.002]$.

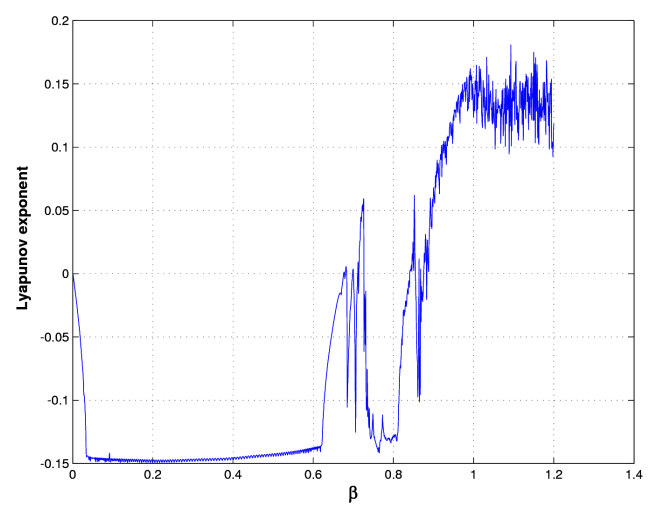

Fig. 6. Lyapunov exponent of uncontrolled evolution of system, where $F=2.5, \gamma=0.3, \omega=\sqrt{3}$, $\alpha=0.8,[x(0), y(0)]=[0.001,0.002]$.

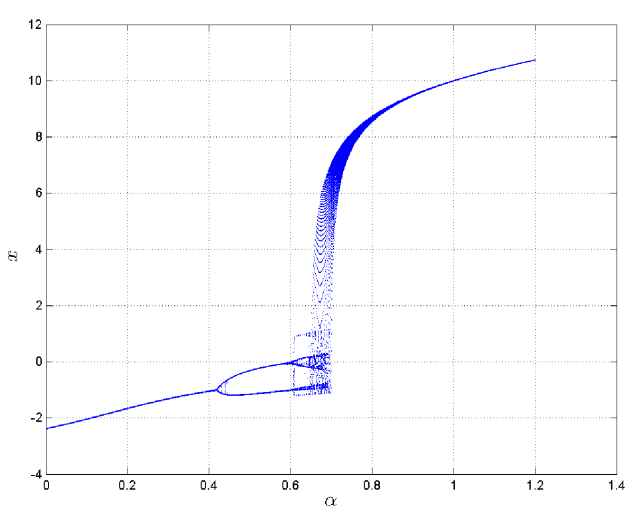

Fig. 7. Bifurcation diagram of uncontrolled evolution of system, where $F=5, \gamma=0.428, \omega=\sqrt{2}$, $\beta=1.12,[x(0), y(0)]=[0.001,0.002]$.

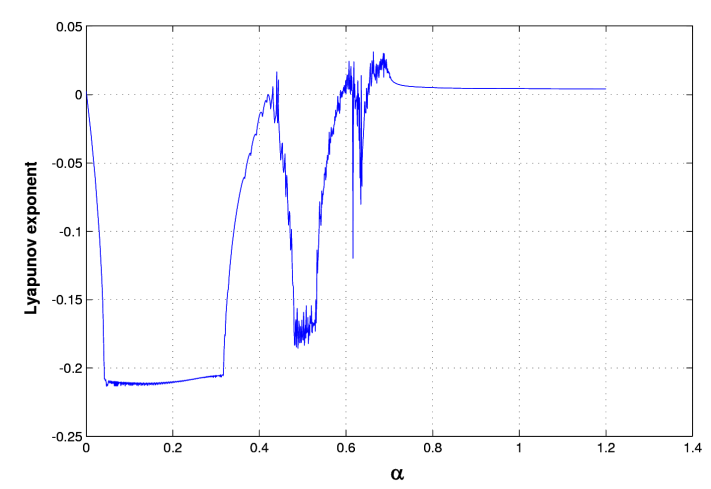

Fig. 8. Lyapunov exponent of uncontrolled evolution of system, where $F=5, \gamma=0.428, \omega=\sqrt{2}$, $\beta=1.12,[x(0), y(0)]=[0.001,0.002]$. 


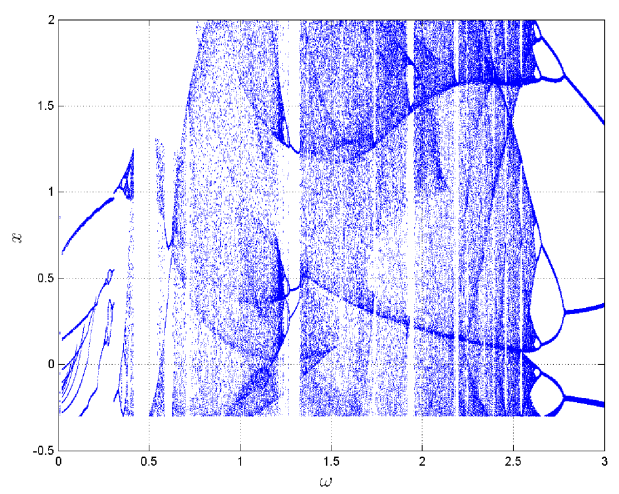

Fig. 9. Bifurcation diagram of uncontrolled evolution of system, where $F=3.3, \gamma=0.8, \alpha=1$, $\beta=8,[x(0), y(0)]=[0,0.5]$.

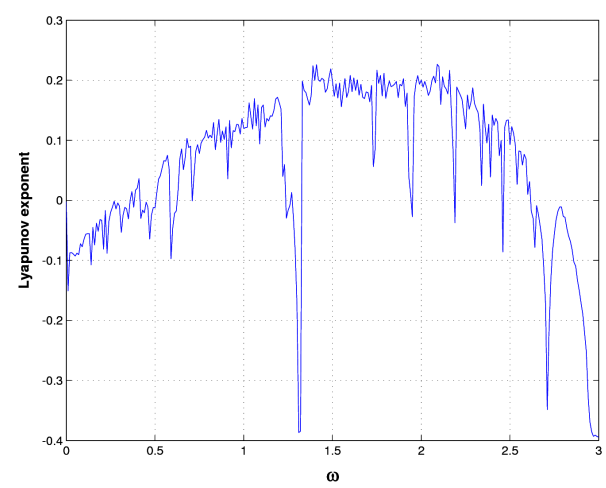

Fig. 10. Lyapunov exponent of uncontrolled evolution of system, where $F=3.3, \gamma=0.8, \alpha=1$, $\beta=8,[x(0), y(0)]=[0,0.5]$.

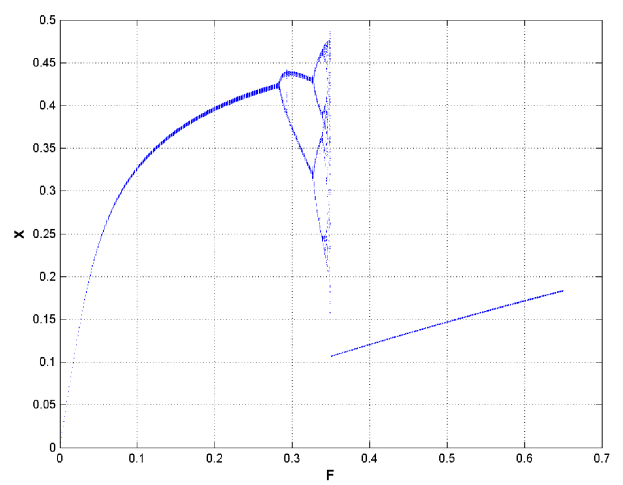

Fig. 11. Bifurcation diagram of controlled evolution of system, where $\gamma=0.16, \beta=1, \omega=\sqrt{1}, \alpha=1$, $\mathrm{d} t=0.001,[x(0), y(0)]=[0.001,0.002]$.

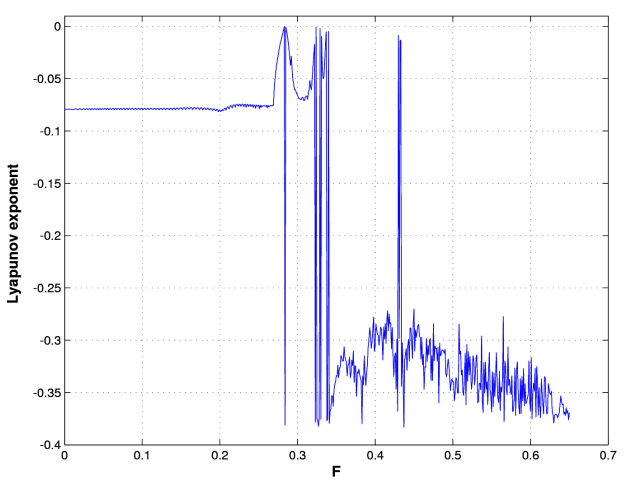

Fig. 12. Lyapunov exponent of controlled evolution of system, where $\gamma=0.16, \beta=1, \omega=\sqrt{1}, \alpha=1$, $\mathrm{d} t=0.001,[x(0), y(0)]=[0.001,0.002]$.

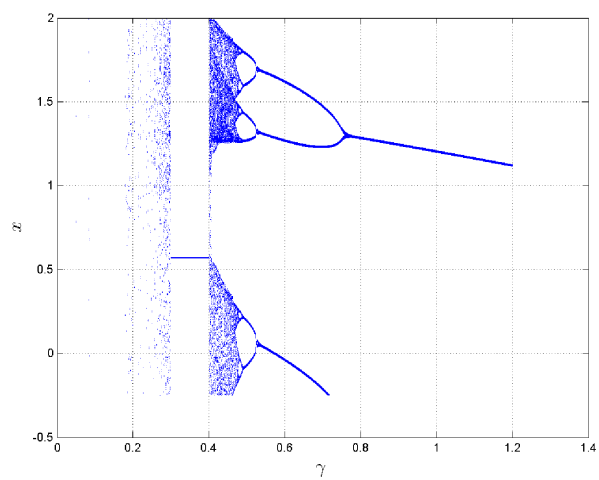

Fig. 13. Bifurcation diagram of controlled evolution of system, where $F=2.5, \beta=0.89, \omega=\sqrt{3}, \alpha=1$, $\mathrm{d} t=0.05,[x(0), y(0)]=[0,0.5]$.

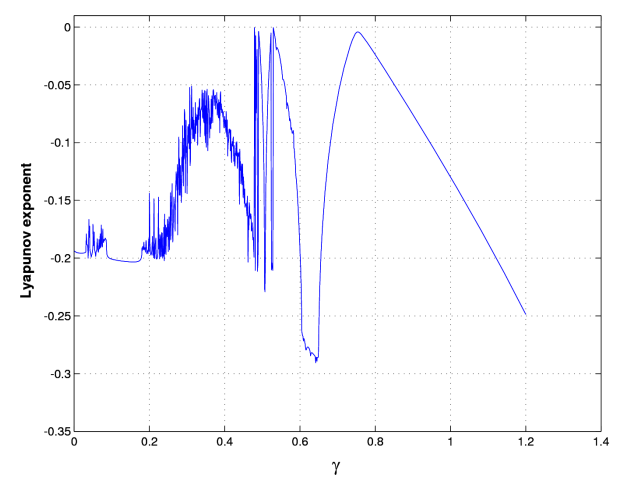

Fig. 14. Lyapunov exponent of controlled evolution of system, where $F=2.5, \beta=0.89, \omega=\sqrt{3}, \alpha=1$, $\mathrm{d} t=0.05,[x(0), y(0)]=[0,0.5]$. 


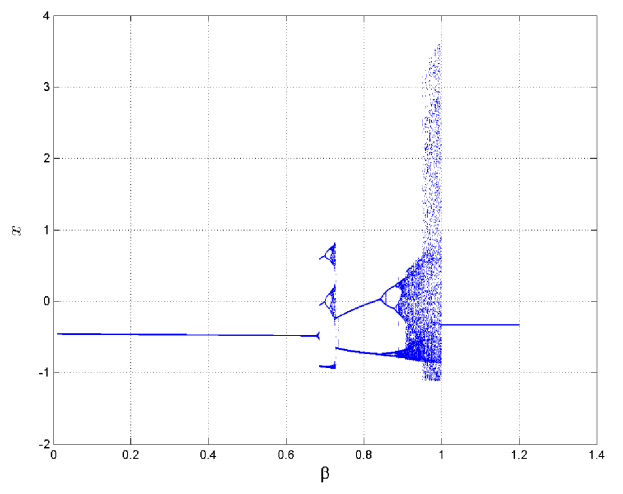

Fig. 15. Bifurcation diagram of controlled evolution of system, where $F=2.5, \gamma=0.3, \omega=\sqrt{3}, \alpha=0.8$, $\mathrm{d} t=0.05,[x(0), y(0)]=[0.001,0.002]$.

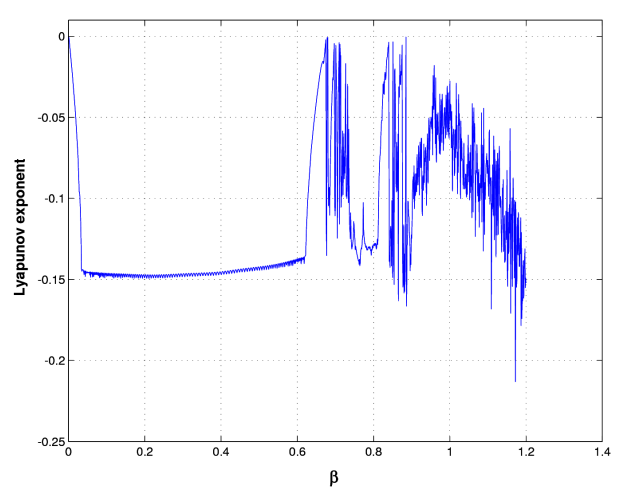

Fig. 16. Lyapunov exponent of controlled evolution of system, where $F=2.5, \gamma=0.3, \omega=\sqrt{3}, \alpha=0.8$, $\mathrm{d} t=0.05,[x(0), y(0)]=[0.001,0.002]$.

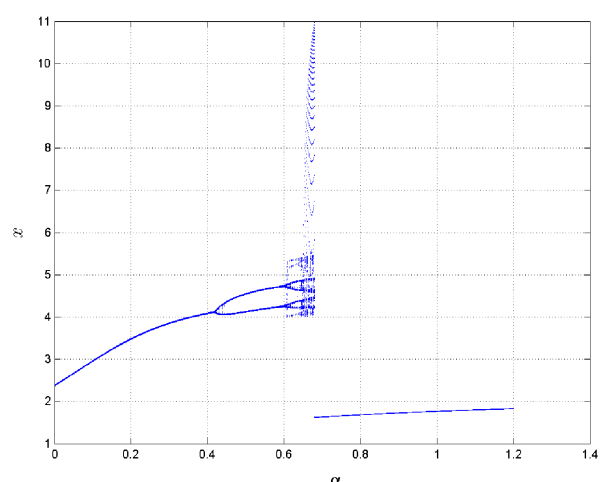

Fig. 17. Bifurcation diagram of controlled evolution of system, where $F=5, \gamma=0.428, \omega=\sqrt{2}, \beta=1.12$, $\mathrm{d} t=0.05,[x(0), y(0)]=[0.001,0.002]$.

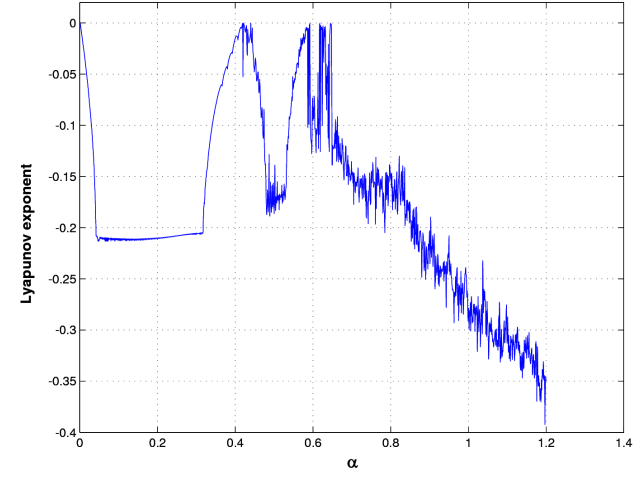

Fig. 18. Lyapunov exponent of controlled evolution of system, where $F=5, \gamma=0.428, \omega=\sqrt{2}, \beta=1.12$, $\mathrm{d} t=0.05,[x(0), y(0)]=[0.001,0.002]$.

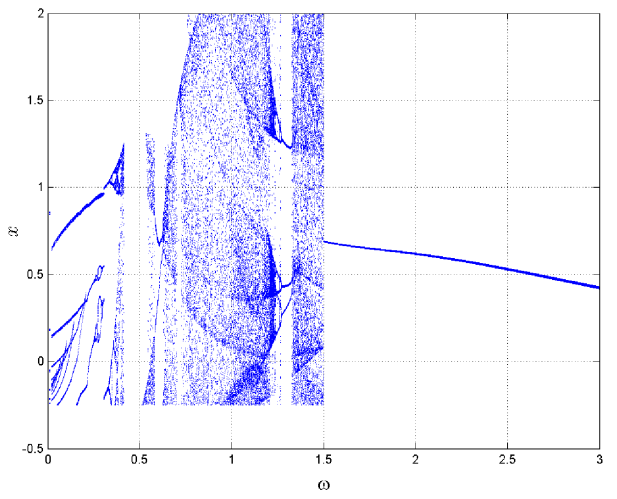

Fig. 19. Bifurcation diagram of controlled evolution of system, where $F=3.3, \gamma=0.8, \alpha=1, \beta=8$, $\mathrm{d} t=0.1,[x(0), y(0)]=[0,0.5]$.

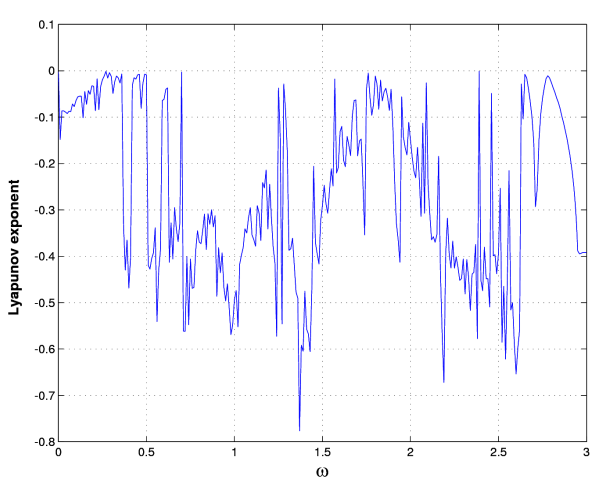

Fig. 20. Lyapunov exponent of controlled evolution of system, where $F=3.3, \gamma=0.8, \alpha=1, \beta=8$, $\mathrm{d} t=0.1,[x(0), y(0)]=[0,0.5]$. 
These figures show that the control process is able to control the chaotic system in its any arbitrary chaotic state.

Although in SMF method Eqs. (5) and (6) can be coupled through any of the control parameters of the system (e.g. $\beta, \alpha, \gamma, F, \omega)$, it is preferable to choose the one that taking the minimum time and this itself depends on the innate of the system [34]. In our study the minimum control time is achieved while coupling two systems through parameter $\gamma$. In fact, the coupling parameter selection is an arbitrary choice [34] and there is no limitation to couple Eqs. (5) and (6) through any of other parameters $\beta, \alpha, F, \omega$.

\section{Conclusion}

The aim of this paper is to develop and apply the SMF control process [34] to continuous nonlinear dynamical systems. In order to examine the impact of the SMF method on the continuous nonlinear systems, the Morse oscillator is used as an example. For over half a century, the Morse potential [42] has been a useful model for the interatomic potential and for fitting the vibrational motion of diatomic molecules, and it is frequently used in theoretical chemistry to describe the photodissociation of molecules [43]. The numerical simulations, including bifurcation diagrams and the Lyapunov exponent plots exhibit the complicated dynamical behaviors of the Morse oscillator.

In the control model based on SMF, the oscillator as the slave system coupled through one of the control parameters with a dynamical system as the master, so its implementation is quite simple. Numerical simulations demonstrate that the proposed process is the most effective way to the control chaos in discrete/continuous dynamical systems. As this method is independent of geometrical considerations, it can easily be applied to high dimensional dynamical systems. The proposed structure can also be carried out to effect the synchronization of chaotic systems. Although the implementation in this paper is performed via numerical simulations, it is not difficult to see that the physical application of the method can be performed. In fact, experimental studies are in progress and the results will be reported elsewhere [44].

\section{References}

[1] G. Dong, From Chaos to Order: Perspectives, Methodologies, Applications, World Sci., Singapore 1998.

[2] A. Saha, P. Wahi, J. Sound Vibrat. 330, 6070 (2011).

[3] K. Ding, Q.L. Han, J. Sound Vibrat. 330, 2419 (2011)

[4] Y.B. Peng, R. Ghanem, J. Li, J. Sound Vibrat. 329, 3660 (2010)

[5] A.N. Njah, U.E. Vincent, J. Sound Vibrat. 319, 41 (2009)

[6] S.Y. Liu, X. Yu, S.J. Zhu, J. Sound Vibrat. 310, 855 (2008).

[7] X. Li, W. Xu, Y. Xiao, J. Sound Vibrat. 314, 526 (2008)

[8] Z. Rahmani, M.R. Jahed Motlagh, Chaos Solitons Fractals 41, 1697 (2009)

[9] H. Salarieh, A. Alasty, Chaos, Solitons, Fractals 41, 67 (2009)
[10] T. Ueta, Int. J. Bifurcat. Chaos. 9, 1465 (1999)

[11] T. Ueta, G. Chen, Int. J. Bifurcat. Chaos. 10, 1917 (2000)

[12] E. Ott, C. Grebogi, J.A. Yorke, Phys. Rev. Lett. 64, 1196 (1990)

[13] C.C. Fuh, P.C. Tung, Phys. Rev. Lett. 75, 2952 (1995).

[14] G. Dong X, IEEE Trans. Circ. Syst. 40, 591 (1993)

[15] M.T. Yassen, Chaos Solitons Fractals 15, 271 (2003)

[16] M.T. Yassen, Chaos Solitons Fractals 26, 913 (2005)

[17] H.N Agiza, Chaos Solitons Fractals 12, 341 (2002)

[18] E.N. Sanchez, J.P. Perez, M. Martinez, G. Chen, Latin Am. Appl. Res: Int. J. 32, 111 (2002).

[19] M.T. Yassen, Appl. Math. Comp. 135, 113 (2001)

[20] T.-L. Liao, S-H. Lin, J. Frankl. Inst. 336, 925 (1999)

[21] J. Lü, S. Zhang, Phys. Lett. A 286, 148 (2001).

[22] J.M. Yuan, in: Directions in Chaos, Ed. B.L. Hao, World Sci., Singapore 1987, p. 164.

[23] R.B. Walker, R.K. Preston, J. Chem. Phys. 67, 2017 (1997)

[24] S.K. Grap, J. Chem. Phys. 75, 67 (1983).

[25] J.R. Ackerhalt, P.O.M. Milonni, Phys. Rev. A 34, 1211 (1986).

[26] P.S. Dardi, S.K. Gray, J. Chem. Phys. 77, 1345 (1982).

[27] F.W. Josellis, J. Differ. Equations 111, 360 (1994)

[28] H. Kobessi, M. Kobessi, C.H. Tard, Can. J. Phys. 72, 80 (1994)

[29] C.G. Lie, J.M. Yuan, J. Chem. Phys. 84, 5486 (1986).

[30] D. Permann, I. Hamilton, J. Chem. Phys. 100, 379 (1994)

[31] K.M. Christoffel, J.M. Bowman, J. Phys. Chem. 85, 2159 (1981)

[32] R. Kapral, M. Schell, S. Fraser, J. Phys. Chem. 86, 2205 (1982)

[33] Z. Jing, J. Deng, J. Yang, Chaos Solitons Fractals 35, 486 (2008)

[34] S. Behnia, A. Akhshani, Chaos Solitons Fractals 42, 2105 (2009)

[35] B.A. Pettitt, J. Chem. Educ. 75, 1170 (1998)

[36] J. Wu, J. Cao, J. Chem. Phys. 115, 5381 (2001)

[37] U. Leonhardt, Phys. Rev. A 55, 3164 (1997)

[38] S. Krempl, T. Eisenhammer, A. Hübler, G. MayerKress, Phys. Rev. Lett. 69, 430 (1992).

[39] M.A. Jafarizadeh, S. Behnia, S. Khorram, H. Nagshara, J. Statist. Phys. 104, 1013 (2001)

[40] S. Boccaletti, C. Grebogi, Y.-C. Lai, H. Mancini, D. Maza, Phys. Rep. 329, 103 (2000).

[41] K. Umeno, Phys. Rev. E 55, 5280 (1997)

[42] P.M. Morse, Phys. Rev. 34, 57 (1929).

[43] M.E. Goggin, P.W. Milonni, Phys. Rev. A 37, 796 (1988)

[44] S. Behnia, S. Afrang, A. Akhshani, Kh. Mabhouti, Opt. - Int. J. Light Electron Opt., in press. 